\title{
Interference Cancellation in Aircraft Cockpit by Adaptive Filters
}

\author{
Arun.C \\ Assistant Professor, Department of ECE, College of Engineering Perumon, Kollam, Kerala, India
}

\begin{abstract}
This paper investigates on the development and implementation of adaptive noise cancellation (ANC) algorithm meant for mitigating the high level engine noise in the cockpit of an aircraft, which makes the speech signal unintelligible. Adaptive filters configured as interference canceller have the potential application in mitigat ing the above interference. A comparative study of Gradient based adaptive Infinite Impulse Response (IIR) algorithm and its modified version is performed using MATLAB simulator in terms of converging speed. From the simulation result the best IIR algorithm is used for implementation in Performance Optimized with Enhanced RISC PC (Power PC) 7448 .
\end{abstract}

\section{Introduction}

High level of engine noise is present in the cockpit of an aircraft. Consider the communication of a pilot by radio from the cockpit to an air traffic controller, the engine noise would be interfered with the speech signal because the noise occupy the same frequency band as speech. The microphone into which pilot speaks also picks the engine noise, which makes the speech signal unintelligible. A conventional filter cannot be used to remove the engine noise because the intensity and frequency of noise components change with engine speed, load and pilot's head. If a second microphone is placed at a convenient location in the cockpit, it is possible to obtain the ambient noise field without pilot's speech. This noise could be filtered and removed from the transmission with the help of adaptive filters configured as noise canceller. There are some situations where a speech signal is interfered by periodic engine noise and no noise field free of pilot's speech is available. In such situations adaptive Line Enhancer (ALE) can be utilized as noise canceller. Th is paper focused on ALE as noise canceller

An adaptive filter is a digital filter that can adjust its coefficients to give the best match to a given desired signal [1]. There are different adaptive algorithms (eg. Least Mean Square (LMS), Normalized Least Mean Square (NLMS) etc) that can be used in time domain. Some widely used methods with Adaptive FIR filter have been explained by S. Haykins [2] \& B.Widrow [3]. Fir filters are more stable than IIR filters. Adaptive IIR filters are attractive: Many fewer coefficients may be needed to achieve the desired performance in some applications [4]. However, it is more difficult to develop stable IIR algorithms, they can converge slowly. Adaptive IIR algorithms are used in some applications (such as low frequency noise cancellation) where the need for IIR-type responses is great. In some cases, the exact algorithm used by a company is a tightly guarded trade secret. However a gradient-based Adaptive IIR algorithm, with some additional features that enable it to adapt more quickly is explained by Don R.Hush [5]. A modified version of the above algorithm gives even faster convergence. So the modified algorithm can be used for implementation.

\section{Adaptive signal processing}

The term Adaptive can be understood by considering a system which is trying to adjust itself so as to respond to some phenomenon that is taking place in its surroundings. This is what adaptation means. Moreover there is a need to have a set of steps or certain procedure called algorith $m$ by which this process of adaptation is carried out [6].

$$
\text { d }
$$

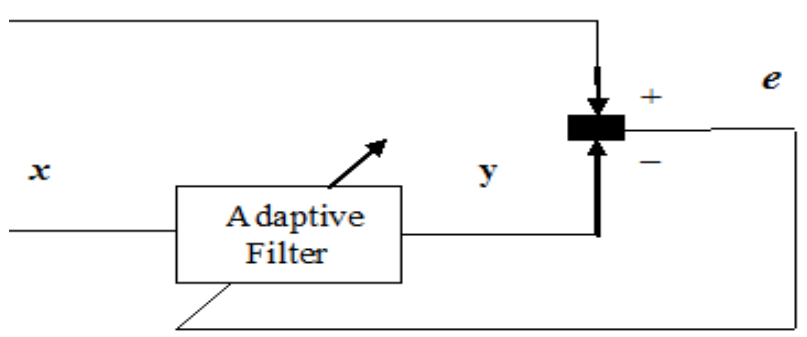

Figure 1. Basic adap tive filter structure

The adaptive filter structure shown in the Figure 1 in which the filter's output $\mathrm{y}$ is compared with a desired signal $d$ to produce an error signal $e$, which is fed back to the adaptive filter. The error signal is given as input to the 
adaptive algorithm, which adjusts the variable filter to satisfy some predetermined rules. In stochastic frame work (based on Wiener filter theory), the optimum coefficients of a linear filter are obtained by minimization of its mean-square error (MSE). All the adaptive algorith ms take the output error of the filter, correlate that with the samples of filter input in some way, and use the result in a recursive equation to adjust the filter coefficients iteratively.

\section{Adaptive noise cancellation (ANC)}

The ANC method uses a "primary" input containing the corrupted signal and a "reference" input containing noise correlated in some unknown way with the primary noise. The reference input is adaptively filtered and subtracted from the primary input to obtain the signal estimate [1].

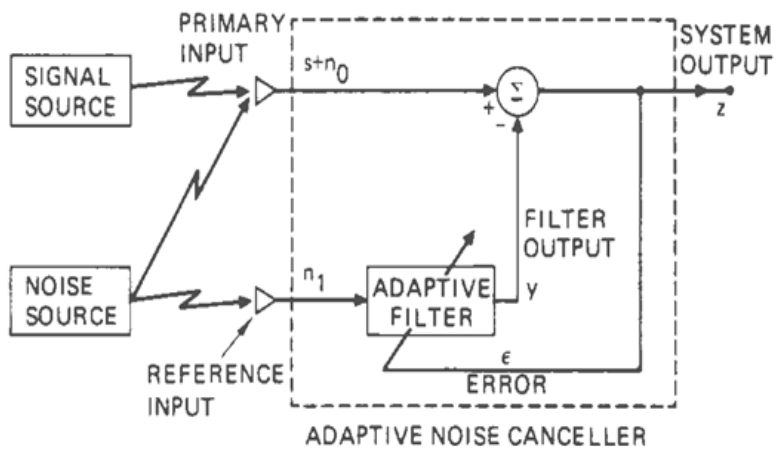

Figure 2. Adaptive noise cancelling concept

A signal $s$ is transmitted over a channel to a sensor that also receives a noise $n_{0}$ uncorrelated with the signal. The combined signal and noise $s+n_{0}$ form the primary input to the canceller. A second sensor receives a noise $n_{1}$ uncorrelated with the signal but correlated in some unknown way with the noise $n_{0}$. This sensor provides the reference input to the canceller. The noise $n_{1}$ is filtered to produce an output $y$ that is as close a replica as possible of $n_{0}$. This output is subtracted from the primary input $s+$ $n_{0}$ to produce the system output $\mathrm{z}=s+n_{0}-y$. Hence the output of the systemcontains the signal alone [1]- [7].

\section{Adaptive line enhancer IIR structure}

The Adaptive Line Enhancer (ALE) enhances the sinusoidal component of the reference input so that output has high signal to noise ratio SNR. From the ALE we also obtain an estimate of the sinusoidal frequency. This ALE can be utilizes as noise canceller. Several forms of the ALE are available. The most popular is the FIR ALE. It has the advantage of being inherently stable and easy to adapt. However, it often requires a large number of filter weights to provide adequate enhancement of narrow-band signals. In an effort to reduce the number of weights, several forms of adaptive recursive filters (IIR) can be used.

The purpose of $\Delta$ is to decorrelate the input noise and its delayed version present at the filter input. This causes the adaptive process to respond only to the sinusoid. As such, H (z) forms a band pass filter around the sinusoid, applying at the same time a phase shift to compensate for the delay so that the sinusoidal component of $\boldsymbol{x}_{\boldsymbol{k}}$ is removed at the summer. At the output of the adaptive filter $x_{k}$, the enhanced sinusoid is obtained. To simplify the analysis assume $n_{k}$ is white noise so that $\Delta=1$ is sufficient for decorrelation.

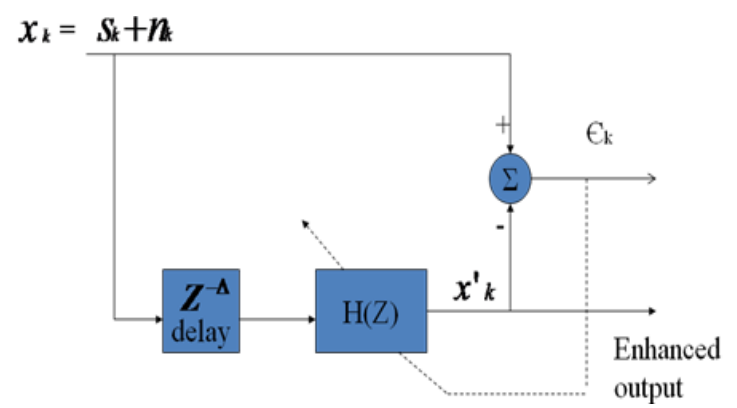

Figure 3. Adaptive line enhancer [2]

\subsection{Gradient based adaptive algorithm for IIR ALE}

The technique used here is a gradient-based algorithm with some additional features that enable it to adapt more quickly. The filter output is given by

$$
\begin{aligned}
& x_{k}^{\prime}=\left\{\frac{1-r^{2}}{1+r^{2}}\right\} w_{k} x_{k-1}-\left(1-r^{2}\right) x_{k-2}+w_{k} x_{k-1}^{\prime}- \\
& r^{2} x_{k-2}^{\prime} \\
& \text { Where } \quad \begin{array}{l}
0<\mathrm{r}<1 \\
-2 \mathrm{r}<\mathrm{w}<2 \mathrm{r}, \quad \mathrm{r} \text { is the radius of the circle }
\end{array}
\end{aligned}
$$

Where just inside the unit circle.

Also from Figure 3, the error output is defined to be

$$
\varepsilon_{k}=x_{k}-x_{k}^{\prime}
$$

The coefficient update for the adaptive algorithm can be expressed as

$$
w_{k+1}=w_{k}+\rho \varepsilon_{k} \alpha_{k}
$$

Where $\rho$ is a parameter which controls the rate of convergence, $\varepsilon_{\mathrm{k}}$ is the error, and $\alpha_{\mathrm{k}}$ is the partial derivative

$$
\alpha_{k}=\frac{\partial x_{k}^{\prime}}{\partial w_{k}}
$$

From (1) and the definition of (3), recursive relationship for

$$
\alpha_{\mathrm{k}} \alpha_{k}=w_{k} \alpha_{k-1}-r^{2} \alpha_{k-2}+\left(\frac{1-r^{2}}{1+r^{2}}\right) x_{k-1}+x_{k-1}^{\prime}
$$

The coefficient update is then defined by (2),(3) and (5).The normalization factor is incorporated by reexpressing the coefficient update in (3) as

$$
w_{k+1}=w_{k}+\mu_{1} \frac{\varepsilon_{k} \alpha_{k}}{\psi_{k}}
$$

Where $\varepsilon_{k}$ and $\alpha_{k}$ are as defined above and 


$$
\psi_{k}=v \psi_{k-1}+(1-v) \alpha_{k}^{2}
$$

The "forgetting factor " $\mathrm{v}$ is in the range $0<<\mathrm{v}<1$

The performance of this algorithm can be enhanced even further by using an approximate partial derivative in place of $\alpha_{k}$ in (5). The modified derivative, denoted by $\alpha_{\mathrm{mk}}$, is obtained from (5) by suppressing the recursive terms, i.e.,

$$
\alpha_{m k}=\left(\frac{1-r^{2}}{1+r^{2}}\right) x_{k-1}+x_{k-1}^{\prime}
$$

\subsection{Simulation results}

After performing the adaptive process, at the output of the filter we get the enhanced version of the sinusoid. At the output of the summer, (error signal) sinusoidal component of input is removed. The simulation result shows the Power Spectral Density (PSD) of pure signal(Figure 4), noise corrupted signal(Figure5), comparisons of PSD of error signals (Figure6)and enhanced signals(Figure7) using both the algorithms. Mean Square Error (MSE) of two algorithms is also shown (Fig ure 8). From the PSD of error signal it is clear that a very large reduction of power occur at the output of the summer. The modified algorithm provides more reduction in power.

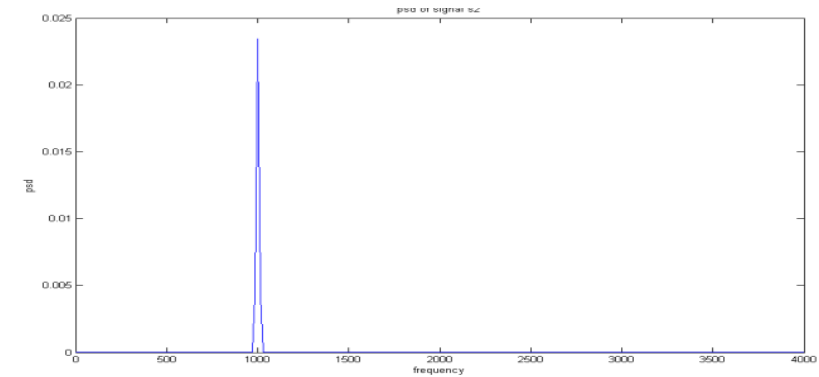

Figure 4. PSD of pure signal

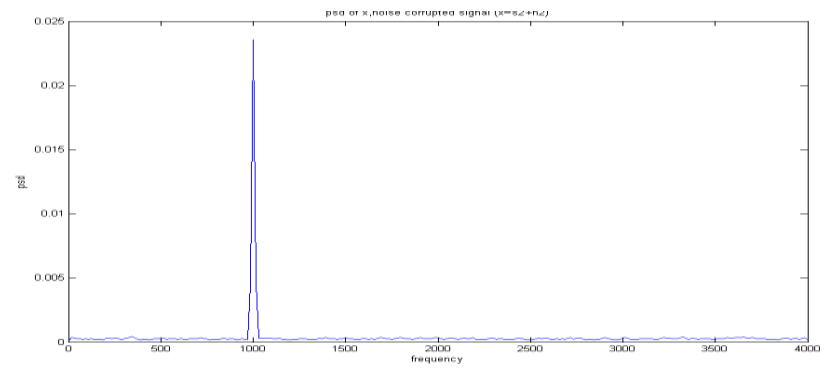

Figure 5. PSD of noise corrupted signal

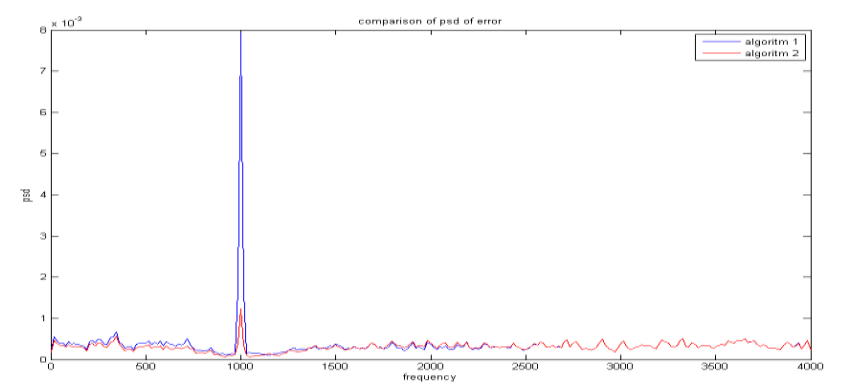

Figure 6. Comparison of PSD of error

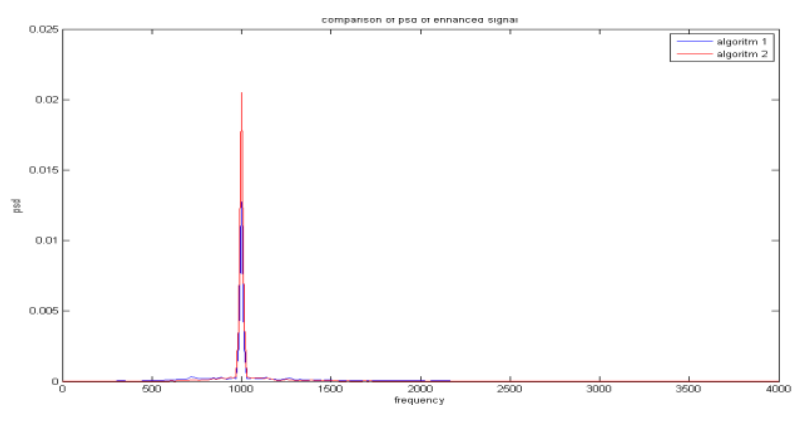

Figure 7. Comparison of PSD of enhanced signal

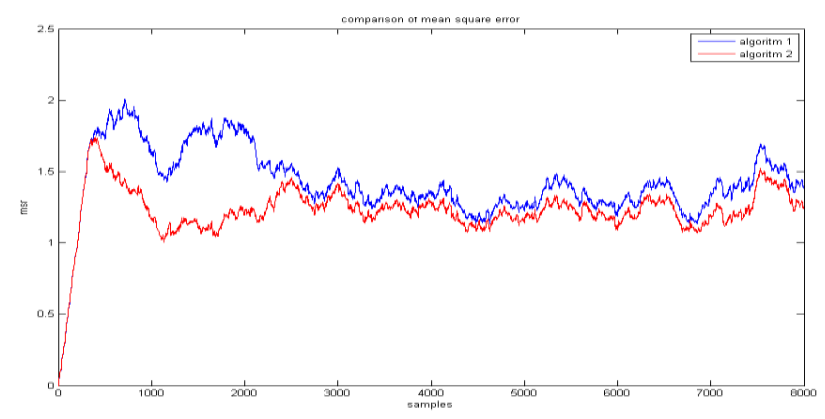

Figure 8. Comparison of mean square error

The Figure 9 shows the convergence time of both the algorithms. Modified algorithm converges faster

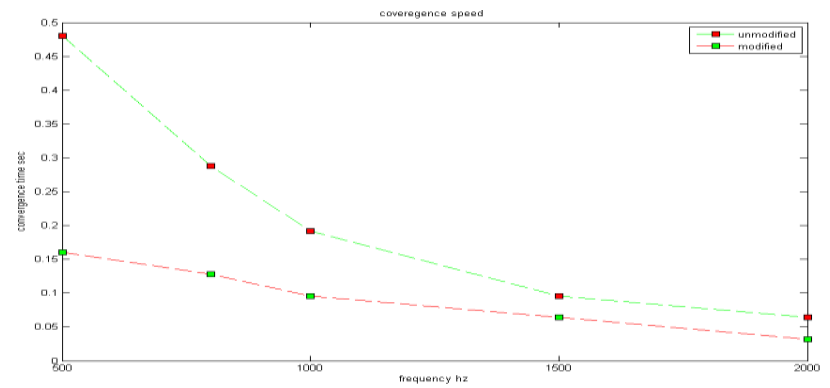

Figure 9. Comparison of convergence time

\section{Adaptive IIR noise cancellation}

ALE can be utilized as noise canceller with experimental data. ALE is used as the basic structure for noise cancellation. Here the input signal $X$ contains the speech signal and engine noise. The purpose of $\Delta$ is to decorrelate the input speech and its delayed version present at the filter input. This causes the adaptive process to respond only to the noise As such, $\mathrm{H}(\mathrm{z})$ forms a band pass filter around the noise, applying at the same time a phase shift to compensate for the delay so that the noise component of $\mathbf{X}$ is removed at the summer. By taking the autocorrelation of speech signal one can find out suitable value for $\Delta$. So at the output of the summer (error signal) contains the speech alone. i.e., the engine noise is adaptively cancelled out. Let the signal to be interfered with noise such that the SNR is controllable and verify the performance of IIR structure in highly noise environment. The system is provided with the 
permission to input a particular SNR (user's choice), a noise with corresponding amplitude is generated and added to signal. This noise corrupted signal will be processed.

\section{$\mathrm{X}=$ Speech + sinusoidal noise}

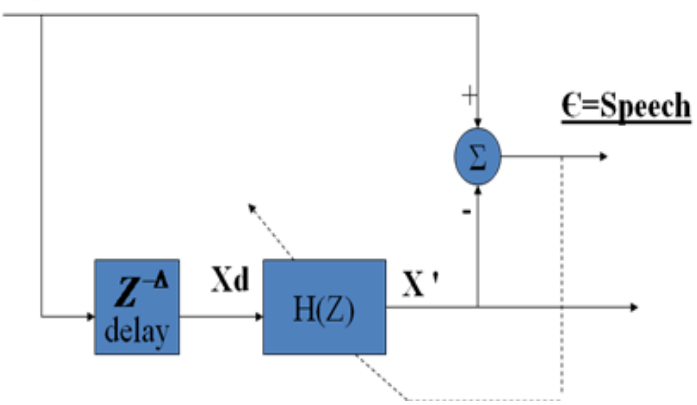

Figure 10. IIR noise canceller

Table 1. SNR and power levels

\begin{tabular}{|c|c|}
\hline SNRin dB & Power level between signal and noise \\
\hline $0 \mathrm{~dB}$ & Signal power $=$ noise power \\
\hline$-3 \mathrm{~dB}$ & Signal power $=(1 / 2)$ noise power \\
\hline$-5 \mathrm{~dB}$ & Signal power $<<$ noise power \\
\hline
\end{tabular}

\subsection{Simulation results}

Verify the performance of algorithm in highly noise environment. Produce the simulation result with SNR = $5 \mathrm{~dB}$, the speech power is too low compared to engine noise.

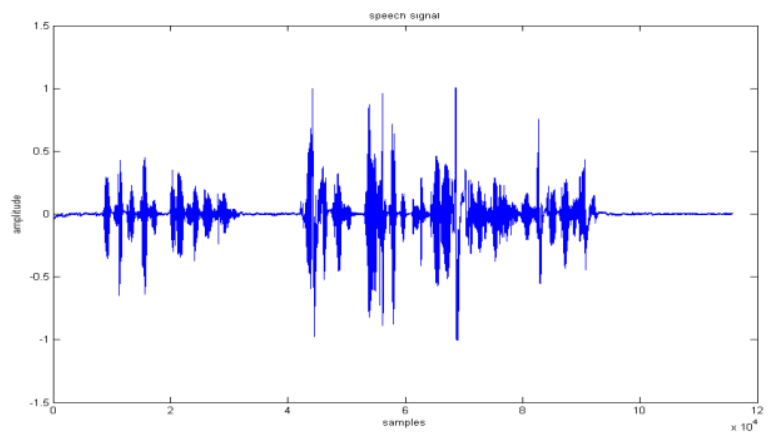

Figure 11. Speech signal

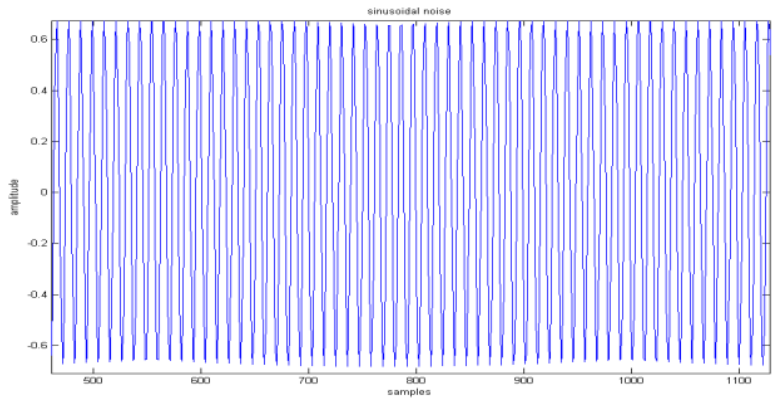

Figure 12. Engine noise

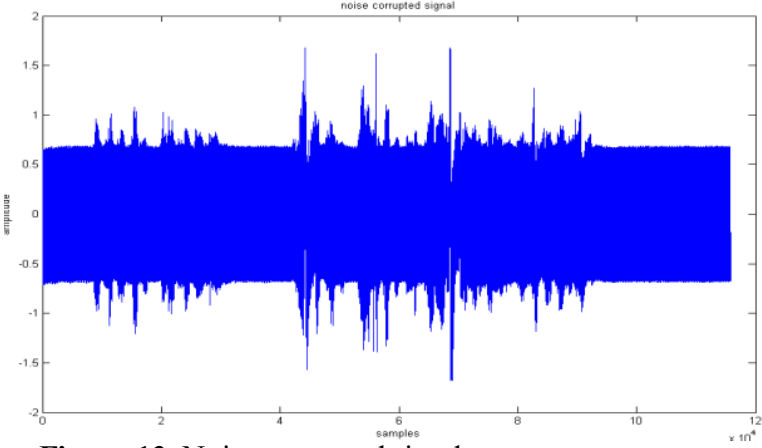

Figure 13. Noise corrupted signal

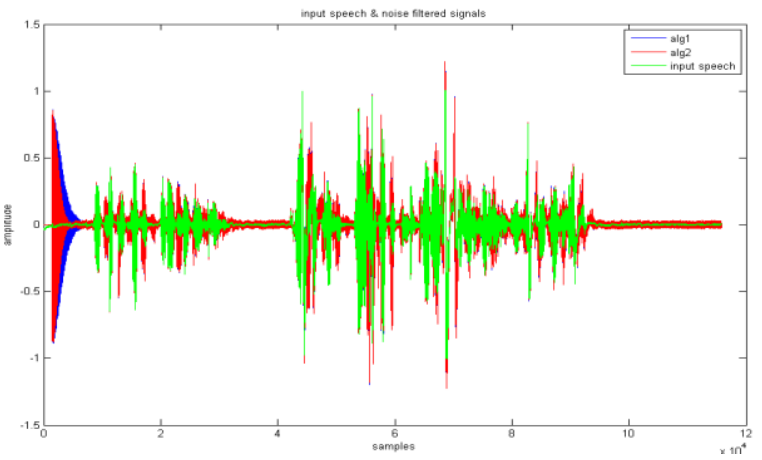

Figure 14. Input speech and noise filtered signals

The Figure11 shows the pure speech and the Figure 12 shows the engine noise. Figure 13 shows the noise corrupted signal. As in ALE here also two adaptive algorithms for noise cancellation were performed, a gradient based algorithm and a modified version of the same.

The Figure14 shows the comparis on among the pure speech and noise filtered signals obtained from two algorith ms. From the simulated result it is clear that even if the signal is interfered by a noise whose power is very high compared to signal power, adaptive IIR filter is able to pick out the noise. The blue colored signal is obtained from normal algorith $\mathrm{m}$. The red colored signal is produced by modified algorithm. From the figure below it is clear that the modified algorithm is converging faster than the normal one. Hence modified version of the gradient based algorithm is used for implementation on PowerPC.

\section{Implementation}

Interference cancellation using adaptive IIR algorithm is implemented in PowerPC 7448 SBC(Single Board Computer).

\subsection{Implementation process flow}

The input signal contains the speech signal interfered by engine noise. The purpose of delay is to decorrelate the input speech and its delayed version present at the filter input. This causes the adaptive process to respond only to the noise. So at the output of the summer (error signal) contains the speech alone. i.e., the engine noise is adaptively cancelled out. 


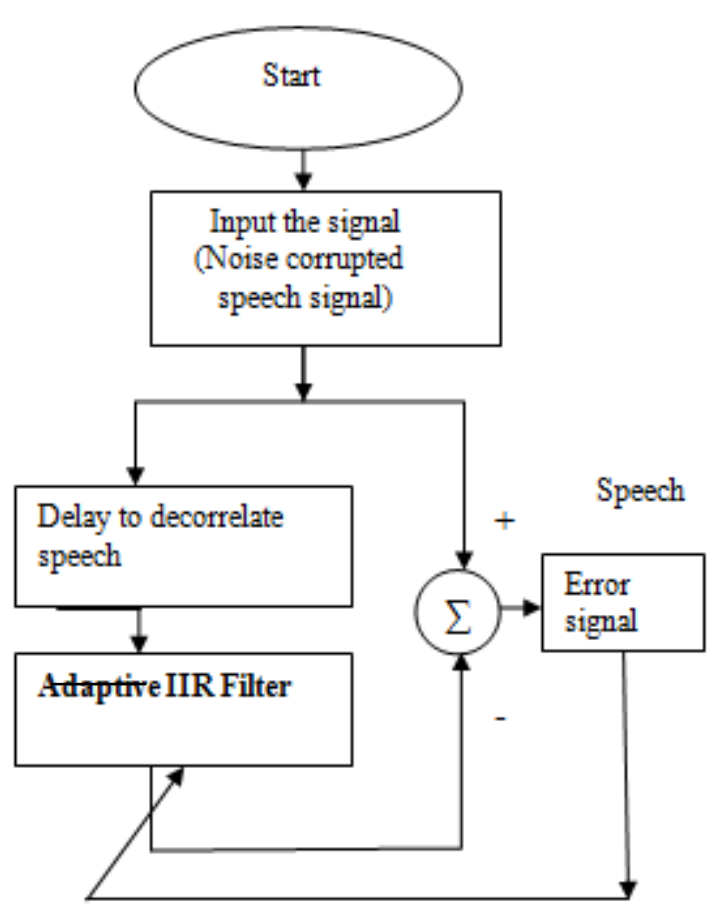

Figure 15. Implementation flow chart

\subsection{Results and discussion}

The interference cancellation using IIR filter has been implemented successfully on PowerPC 7448 with Modified Gradient Based Adaptive algorithm and verified the output. Here the signal applied to the input is speech corrupted by engine noise. After adaptive filtering process speech alone is produced at the output.

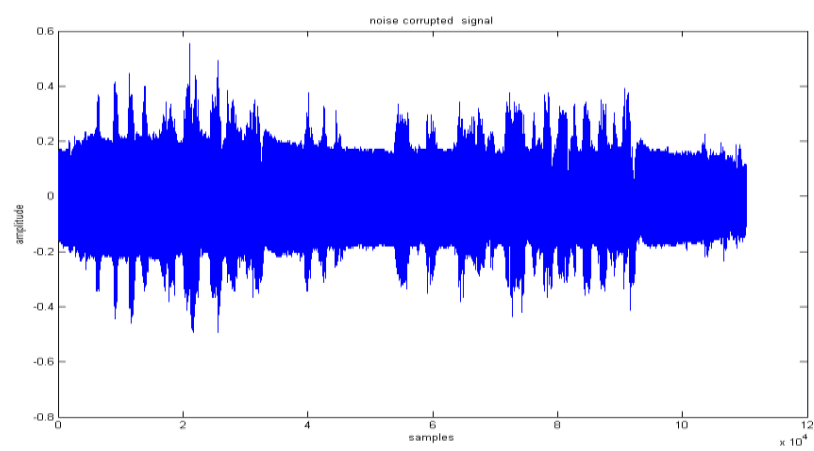

Figure 16. Noise corrupted speech signal

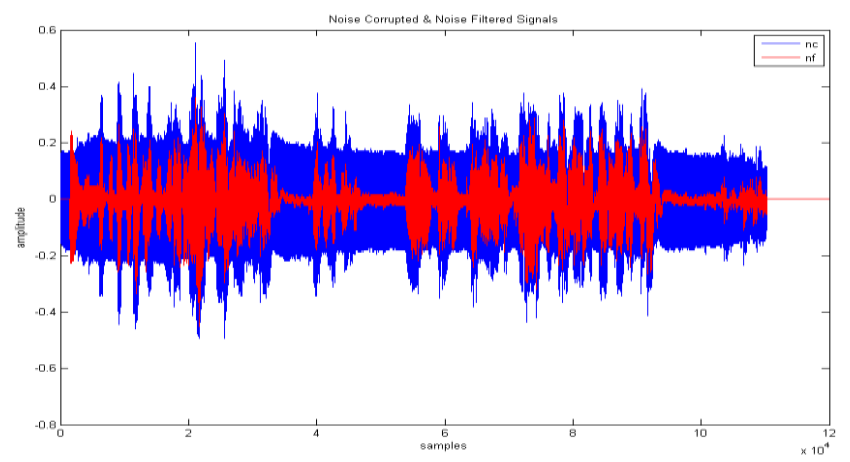

Figure 17. Noise corrupted and noise filtered speech signals
Figure 17 show the noise corrupted signal and noise filtered signal together. The Adaptive filter filtered the engine noise and produced the speech alone at the output.

\section{Conclusion and future scope}

A detailed survey on ANC is carried out and observed that many fewer coefficients may be needed to achieve the desired performance for IIR filter when compared to FIR. From simulation under different noise environment, it is concluded that the modified gradient based algorithm is more suitable for Adaptive IIR noise cancellation. Even if the signal is interfered by a noise whose power is very high compared to signal power, adaptive IIR filter is able to pick out the noise. i.e., even in the highly noise environment adaptive IIR filter is powerful.

Future work includes the implementation of the pipelined version of the filter for improved speed, and to optimize the programmable processing element at circuit level for efficient ASIC implementation of the reconfigurable fabric.

\section{References}

1. B. Widrow et al., "Adaptive noise cancelling: Principles and applications," Proc. IEEE, vol. 63, pp. 1692-1716, Dec. (1975).

2. S. Haykin: Adaptive Filter Theory, Englewood Cliffs, N.J.: Prentice-Hall, Inc., $4^{\text {th }}$ Edition (2001).

3. B. Widrow and S. Stearns: Adaptive Signal Processing. Prentice Hall, p. 301(1985).

4. Bijan Sayyarrodsari et al.,"An Estimation-Based Approach to the design of Adaptive IIR Filters", Proceedings of the American Control Conference Philadelphia, Pennsylvania 1 June (1998).

5. Don R. Hush et al., "An Adaptive IIR Structure for sinusoidal Enhancement, Frequency Estimation and Detection", IEEE Trans. Acoust., Speech, Signal Processing, vol.ASSP-34, No.6, pp.1380-1390, $\operatorname{Dec}(1986)$.

6. J.J. Shynk. Adaptive IIR filtering. IEEE ASSP Magazine, Vol. 6, No. 2, pp. 4-21, April (1989).

7. M. Harteneck, R.W Stewart, "Adaptive Digital Signal Processing Java Teaching Tool" Submitted to IEEE Transactions on Education - Special CDROM Is sue, November (1999).

8. Farhang-Boroujeny,B. Adaptive filters: theory and applications, John Wiley\& sons Ltd,(1998).

9. Freescale semiconductor, "MPC 7448 RISC Microprocessor Hardware Specifications" a 60 page PDF manual, (2005).

10. O.L Frost III ,"An algorith $m$ for linearly constrained adaptive array processing"Proc.IEEE,vol.60.pp.926$935 \operatorname{Aug}(1972)$.

11. Adaptive Filter Theory by Simen Haykin: 3rd edition,Pears on Education Asia.LPE 